\title{
Spin transparency mode in the NICA collider
}

\author{
Yury Filatov ${ }^{1}$, Alexander Kovalenko ${ }^{2, *}$, Andrey Butenko ${ }^{2}$, Evgeniy Syresin ${ }^{2}$, \\ Vladimir Mikhailov ${ }^{2}$, Stepan Shimanskiy ${ }^{2}$, Anatoliy Kondratenko ${ }^{3}$, and \\ Mikhail Kondratenko ${ }^{3}$ \\ ${ }^{1}$ Moscow Institute of Physics and Technology, Dolgoprudny, Russia \\ ${ }^{2}$ Joint Institute for Nuclear Research, Dubna, Russia \\ ${ }^{3}$ Science and Technique Laboratory "Zaryad", Novosibirsk, Russia
}

\begin{abstract}
The NICA collider can operate with polarized light ions in two modes. At the Preferred Spin mode (PS mode) the periodic spin motion along the closed orbit is unique, i.e. the static magnetic lattice determines a single stable orientation of the beam polarization at any collider's place. At the Spin Transparency mode (ST mode) any spin direction repeats every particle turn along the closed orbit, i.e. the collider's magnetic lattice is transparent to the spin. ST mode allows one to use a completely new approach to carry out experiments with polarized ions at high precision level. The features of ion polarization control in the ST mode are discussed. The schemes of polarization control in the NICA collider in the ST mode are presented.
\end{abstract}

\section{Spin transparency and preferred spin modes in the NICA collider}

In the NICA collider the polarization of protons and deuterons can be controlled in two modes: in Preferred Spin (PS) mode, when the collider's structure determines a single stable polarization direction, and in Spin Transparency (ST) mode, when any polarization direction is repeated after particle's each turn $[1,2]$. The main difference between the PS and ST modes appears at the manipulations of the polarization direction during an experiment. In the ST mode the spin motion is very sensitive to any small perturbations of the magnetic field, because particles are in the region of the integer spin resonance. To stabilize the required polarization direction in the SPD or MPD detectors or at any specified point of the collider, it is sufficient to use "weak" magnetic fields which rotate the spins at small angles $\Psi \ll 1$. To control ion polarization in the NICA collider over the momentum range up to $13,5 \mathrm{GeV} / \mathrm{c}$, it is preferable to use a pair of weak solenoids with field integrals of $\sim 1 \mathrm{~T} \cdot \mathrm{m}$ which do not perturb the closed orbit [3].

To change polarization direction in the PS mode it is required to use spin rotators based on a strong fields, rotating the spins at the angles of $\Psi \sim 1$. To rotate the polarization from the vertical direction to the longitudinal one, conventionally it would be necessary to apply the transverse fields with the total integral of 20-30 T.m that leads to a strong distortion of the particle's closed orbit, which can reach of tens of centimeters at low energies [4]. Using longitudinal strong fields leads to spin resonances crossing with beam depolarization during manipulation of polarization. Thus, efficient polarization control of ions, deuterons

\footnotetext{
*e-mail: kovalen@dubna.ru
} 
a)

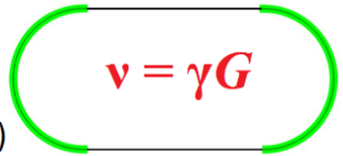

b)

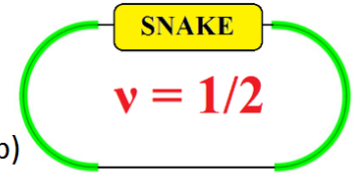

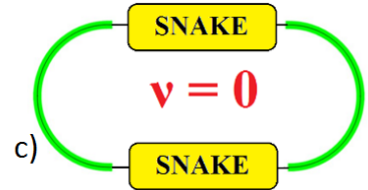

Figure 1. Possible operating regimes with polarized ions: a) the NICA collider without snakes, b) the NICA collider with one snake, c) the NICA collider with two snakes

Table 1. Polarization in the SPD and MPD detectors in PS and ST modes

\begin{tabular}{llll}
\hline $\begin{array}{l}\text { Collider's } \\
\text { configuration }\end{array}$ & $\begin{array}{l}\text { Spin } \\
\text { tune }\end{array}$ & $\begin{array}{l}\text { Spin } \\
\text { mode }\end{array}$ & $\begin{array}{l}\text { Polarization direction } \\
\text { at SPD/MPD detector }\end{array}$ \\
\hline Without snakes & $\gamma G \neq k$ & PS & Vertical at SPD and MPD \\
Without snakes & $\gamma G=k$ & ST & Any direction at SPD or MPD \\
One snake (SPD) & $1 / 2$ & PS & Longitudinal at MPD \\
One snake (MPD) & $1 / 2$ & PS & Longitudinal at SPD \\
Two snakes & 0 & ST & Any direction at SPD or MPD \\
\hline
\end{tabular}

especially, in the NICA collider by means of quasi-stationary weak fields is possible the only if the ST mode is used.

The configuration of the NICA collider with two solenoid snakes inserted into its straights allows one to operate with polarized proton and deuteron beams in both PS and ST modes (see Fig. 1, Tab. 1).

Stable polarization direction is vertical at any point of the orbit in the NICA collider without snakes, whereas the spin tune is proportional to the particle energy: $v=\gamma G$, where $G$ is an anomalous part of the gyromagnetic ratio. The collider operates in the PS mode practically over the total energy range then $\gamma \neq k / G$, where $k$ is an integer. At integer spin resonances $\gamma G=k$ the collider operates in the ST mode which gives an opportunity to have any direction of the polarization in the both detectors.

After switching one of the snakes on, the collider will operate in PS mode with the spin tune equal to half. The snake transforms completely the spin motion providing a stable longitudinal direction of the polarization in opposite respect to the snake straight.

If two snakes are switched on, the ST mode is realized at any energy. The spin tune does not depend on particle energy and is equal to zero that gives capability to obtain any direction of the polarization at any point of the collider orbit.

\section{Polarization manipulation in the NICA collider in ST mode}

\subsection{ST-mode at integer spin resonances}

In the NICA collider without snakes, the ST mode is realized at discrete energy points corresponding to integer spin resonances: $\gamma=k / G$. For protons the number of such points is of 25 starting from minimal energy $E_{k i n}^{\min }=108 \mathrm{MeV}$ with the step of $\Delta E=523 \mathrm{MeV}$. For deuterons there is only one point with $E_{k i n}=5,63 \mathrm{GeV} / \mathrm{u}$.

A possible scheme of ion polarization control in the ST mode at the integer spin resonances is presented in Fig. 2. Two polarization control (PC) insertions symmetrically placed around MPD are used to stabilize the required polarization direction at any place of the collider ring, including the interaction points, injection points, etc. In each PC insertion two 


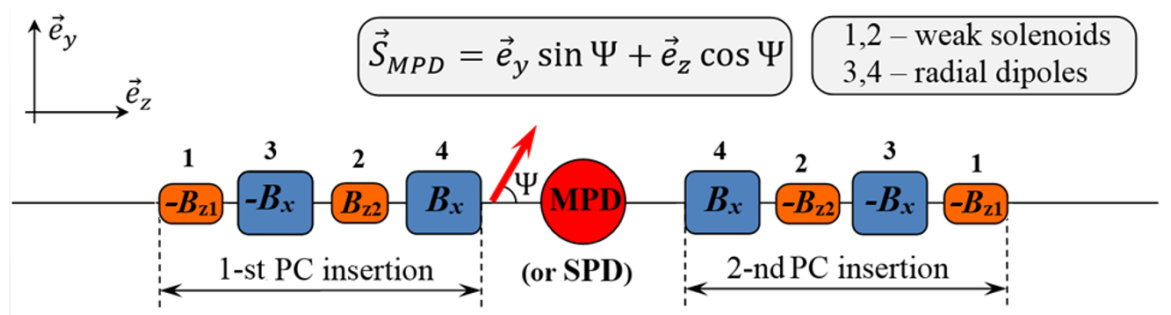

Figure 2. Scheme of the polarization control (PC) insertions in the NICA collider's ST mode

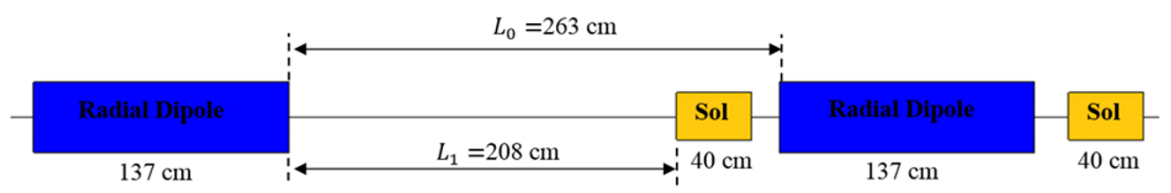

Figure 3. Placement of weak control solenoids among structural radial dipoles

weak solenoids with longitudinal field $B_{z 1}$ and $B_{z 2}$ are placed around the structural dipoles with radial field $B_{x}$, providing deflection of the beams to the collision plane of the MPD.

The scheme makes it possible the ion polarization control in the vertical plane $(y z)$ in MPD or SPD ( $\Psi$ is the angle between polarization and particle velocity vectors). The value of the control weak solenoids is limited by the polarization stability condition: one must ensure that the spin tune induced by the solenoids significantly exceeds the strength of the integer spin resonance, determined by the orbital emittances and errors of imperfections. The scheme provides polarization control for all discrete points over NICA energy range if the field integral will reaches $0,6 \mathrm{~T} \cdot \mathrm{m}$ in each of four solenoids. The control solenoid length is of $40 \mathrm{~cm}$ if we limit the maximum field to $1,5 \mathrm{~T}$. The placement of weak solenoids among radial dipoles in the real relative scale is shown in Fig. 3.

\subsection{ST mode by means of two snakes}

To provide ST mode in the NICA collider at any energy two solenoidal snakes are installed symmetrically in respect to both MPD and SPD setups (Fig. 4). The configuration makes it possible to rotate the polarization in the vertical plane (yz) of MPD or SPD detector, whereas in the collider magnet arcs the polarization vector is moving in the median plane $(x z)$ [5].

At the maximum momentum of $13,5 \mathrm{GeV} / \mathrm{c}$ the total integral of longitudinal solenoidal field per half-snake should reach of $25 \mathrm{~T} \cdot \mathrm{m}$ for protons and of $80 \mathrm{~T} \cdot \mathrm{m}$ for deuterons respectively. One can distribute short solenoids along the experimental straights to form the snakes. In the case of $6 \mathrm{~T}$ solenoids the total length of proton half-snake is of $4,2 \mathrm{~m}$.

It is possible to adopt the collider lattice structure optimized for heavy ion beam for the case of the ST mode at the protons mode over the total energy range. Weak control solenoids do not disturb practically the orbital motion in the collider where strong solenoids of the snakes lead to a strong betatron tunes coupling. Since the longitudinal field of the snakes changes proportionally to the particle's momentum, the orbital characteristics do not depend on the collider's energy. Matching of the solenoids with the collider structure is provided by means of proper choice of the work point by means of structural $K F$ (focusing) and $K D$ 


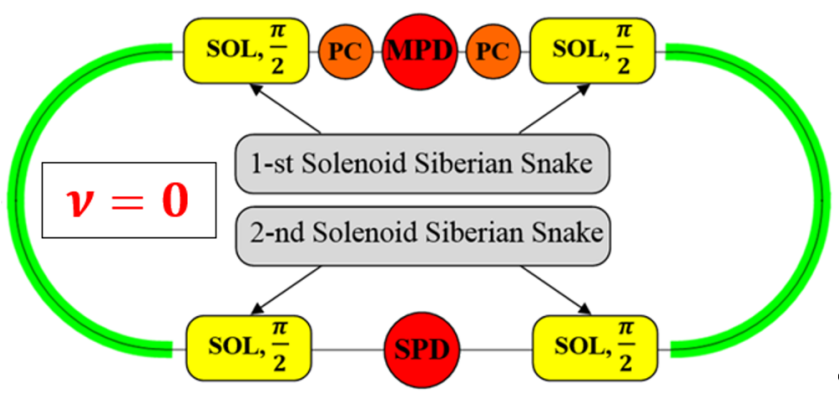

Figure 4. Polarization control in the NICA collider with two solenoid snakes (the ST mode over the total energy range)

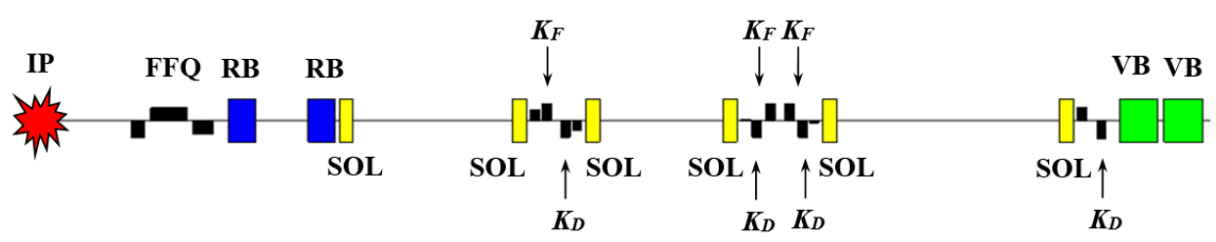

Figure 5. Distributed snake (one half) based on short 6 T SC solenoids

(defocusing) quadrupole lenses. A possible scheme of the distributed snake (one half) based on short $6 \mathrm{~T}$ superconducting solenoids (SC) is shown in Fig. 5.

The elements are the following: $S O L$ is $\mathrm{SC}$ solenoid, $F F Q$ is the final focus triplet of the collider, $V B$ are structural dipole magnets, $R B$ are bending dipoles with transverse field for converging the bunches in the collision point $I P$.

\section{ST modes in the NICA collider without and with snakes}

Snakes provide independence of the spin tune on energy that excludes the impact of synchrotron oscillations and higher-order spin resonances on the polarization. The polarization stability at long times (up to tens of hours) has been demonstrated in the collider RHIC (BNL, USA), in which the independence of the spin tune on energy is provided by two helical snakes [4]. At the operating in the NICA collider without snakes, the spin tune is proportional to the energy and the synchrotron oscillations lead to a spin tune spread, which can have a strong influence on the spin dynamics. There is a problem of finding the energy regions, in which the polarization lifetime is greater than the time of beam storage.

When collider is operating in the ST mode on integer spin resonances, the main problems to preserve polarization during beam acceleration to the energy of experiment is transferred to the Nuclotron. To preserve the polarization of protons in the whole energy range, a $25 \%$ solenoid snake with field integral $\sim 12 \mathrm{~T} \cdot \mathrm{m}$ can be used in the Nuclotron that requires a substantial modernization of the Nuclotron [6].

When the collider is operating in the ST mode with snakes, it is sufficient to inject protons at the minimum collider's energy with further acceleration of the polarized beam to the energy of experiment. To preserve the proton polarization in the Nuclotron up to $2,5 \mathrm{GeV}$, which corresponds to the first intrinsic spin resonance, it suffices to use a $1 \%$-solenoidal snake with an integral field of $\sim 0,5 \mathrm{~T} \cdot \mathrm{m}$ that does not require significant modernization of the Nuclotron. 
The problem of reaching the highest possible luminosity of polarized proton collisions is connected with the particle multi-bunch storage in the collider and electron cooling of the stored beam during the process. The optimal proton beam kinetic energy at the beam injection into the collider is about $1 \mathrm{GeV}$. Thus, to obtain polarized beams with high luminosity, it is necessary to use only the NICA collider with snakes.

\section{Spin flipping system and system of on-line polarimetry}

The Spin-Flipping (SF) system is of particular interest for the experiment. The SF system allows one to simultaneously reverse the polarization of all bunches during the experiment, without changing the orbital characteristics of the beam. The SF system based on quasistationary fields is naturally realized in the ST collider mode. The pair of the weak solenoids provides simultaneous control of the polarization direction and the spin tune. Thus, the opportunity of the spin tune stabilization during the spin flipping takes place, preventing both as the zero and as higher order spin resonances crossing. The polarization degree will be kept with an exponential accuracy, if the field of the weak control solenoids is changed slowly. For realization of the SF system in the collider operating in a PS mode it would be necessary to introduce in the lattice RF-field of a MHz's range with the field total integral of $1 \mathrm{~T} \cdot \mathrm{m}$, that is not an easy technical task and requires detailed theoretical and experimental researches [7].

To analyze the asymmetry in the scattering of polarized particles in the PS mode, it is necessary, as it is done in the RHIC collider, to use a sequence of bunches, which is formed directly from a source of polarized protons, with alternating polarization directions in the bunches. However, at the detector the same pairs of bunches will always collide with fixed polarization directions. One can get information about the asymmetry of scattering the polarized particles only by comparing the results of scatterings that occurs in other pairs of bunches.

The SF system in the ST mode provides a simultaneous spin reversals in all bunches of one ring. As a result, the same bunches will collide in the detector, whose the polarization direction can be alternately changed. In this approach, polarization reversal during formation of the sequence of bunches from the source of polarized ions is not required. It is enough to inject into the collider "identical" bunches with the same polarization direction. Thus, the measurement of the bunch-to-bunch luminosity and development of the system of bunches monitoring are not required. It becomes possible to compare the bunches not only with the opposite polarization direction, but also with any desired directions: vertical-longitudinal, vertical-radial, radial-longitudinal, etc.

Also in the ST mode, the unique possibility of the on-line polarization monitoring (online polarimetry) takes place. Due to the field variation time in weak solenoids $(\sim 0,1-1 \mathrm{~s})$ is much larger of the spin precession period around the induced spin field ( $\sim 0,1-10 \mathrm{~ms})$, any manipulations with the spin direction will happen adiabatically and the polarization degree during the experiment time will be supported permanently. Then the vector of polarization will be a function of the weak solenoids field and can be monitored by means of the solenoid field measurements.

Both systems of spin-flipping and on-line polarimetry makes it possible to carry out the experiments at the NICA collider at a new level of the accuracy.

\section{Summary}

Tab. 2 presents the summarized information concerning possibilities at the operation with polarized protons and deuterons for the following collider's configurations: NICA without 
Table 2. Possibilities at the operation with polarized protons and deuterons in the NICA collider

\begin{tabular}{llllll}
\hline $\begin{array}{l}\text { Collider's } \\
\text { configuration }\end{array}$ & $\begin{array}{l}\text { Spin } \\
\text { mode }\end{array}$ & $\begin{array}{l}\text { SF } \\
\text { system }\end{array}$ & $\begin{array}{l}\text { On-line } \\
\text { polarimetry }\end{array}$ & $\begin{array}{l}\text { Scanning } \\
\text { of energy }\end{array}$ & $\begin{array}{l}\text { Impact synchrotron } \\
\text { oscillations on spin }\end{array}$ \\
\hline without snakes & PS & No & No & No & Impact \\
without snakes & ST & Yes & Yes & No & Impact \\
with one snake & PS & No & No & Yes & Doesn't impact \\
with two snakes & ST & Yes & Yes & Yes & Doesn't impact \\
\hline
\end{tabular}

snakes and NICA with one or two snakes. In addition to the possibilities of implementing systems of spin-flipping and on-line polarimetry, and the possibility of carrying out experiments at any collider's energy (scanning of energy), the table also gives information about the synchrotron oscillations influence on the beam polarization. The independence of the spin tune on the energy and very small spin tune spread in the NICA collider with one and two snakes allows one to significantly stabilize the spin dynamics and greatly increase the lifetime of polarized beams compared to the NICA collider without snakes.

\section{Conclusion}

In conclusion, let us summarize the main results presented in this paper.

- The configuration of the NICA collider with solenoid snakes significantly expands the possibilities of carrying out experiments with polarized protons and deuterons, and allows one to compare experimentally different polarization control modes.

- Using of solenoid snakes provides the independence of the orbital and spin characteristics of the collider during the energy change.

- To perform the spin physics program at JINR, it is necessary to use the spin transparency mode in the NICA collider with two snakes, which allows one to apply a completely new approach to carry out experiments with polarized ions at the high precision level.

- The presented universal scheme of polarization control in ST mode allows to operate with the polarization of any particle species, including deuterons.

\section{References}

[1] A.D. Kovalenko et al., Physics of Particles and Nuclei 45(1), 325-326 (2014)

[2] A.D. Kovalenko et al., J. of Phys.: Conf. Series 938(1), 012025 (2017)

[3] Yu.N. Filatov et al., in Proc. of XV Advanced Research Workshop on High Energy Spin Physics (DSPIN-13), (Dubna, JINR, 2014) 351-354

[4] V.I. Ptitsyn, Yu.M. Shatunov, NIM A 398, 126 (1997)

[5] A.D. Kovalenko et al., in Proc. of IPAC'15, JACoW Publication, Richmond, VA, USA, paper TUPTY017, 2031-2033 (2015)

[6] Yu.N. Filatov et al., in Proc. of IPAC'17, JACoW Publication, Copenhagen, Denmark, paper TUPVA112, 2349 (2017)

[7] Y.S. Derbenev et al., in Proc. of XIV Advanced Research Workshop on High Energy Spin Physics (DSPIN-11), (Dubna, JINR, 2012) 377-384 